\title{
Analysis of Microbial Composition Associated with Freshwater and Seawater
}

\author{
So-Yeon Lee ${ }^{1}$ and Yong-Bin Eom ${ }^{1,2, \uparrow}$ \\ ${ }^{I}$ Department of Medical Science, College of Medical Sciences, Soonchunhyang University \\ Asan, Chungnam 31538, Korea \\ ${ }^{2}$ Department of Biomedical Laboratory Science, College of Medical Sciences, \\ Soonchunhyang University, Asan, Chungnam 31538, Korea
}

\begin{abstract}
Knowledge of the distribution and biodiversity of environmental bacteria and the ecosystem that influences them is crucial for predicting an ecosystem. However, bacterial culture methods can only analyze approximately $0.1 \%$ of the existing microorganisms, those that are readily cultured under laboratory conditions. By contrast, next-generation sequencing (NGS) has generally been known to obtain more diverse profiling of bacterial composition. We compared the bacterial communities using both a culture-dependent (MALDI-TOF) and culture-independent (NGS) methods. Environmental specimens were obtained from both freshwater and seawater. Water samples were also analyzed by both pyrosequencing and MiSeq sequencing, in order to select one NGS platform which could analyze comparatively more diverse microbiota. Bacterial distribution analyzed with MALDI-TOF showed no difference between the microbiota of freshwater and seawater, whereas the results analyzed with NGS distinguished between the two. The diversity indexes of MiSeq sequencing were higher than for Pyrosequencing. This indicated that MiSeq sequencing is capable of analyzing a comparatively wider diversity of bacteria. The genus of Flavobacterium and Planktophila were identified as being unique to freshwater, whereas EU801223 and OM43 were found in the seawater. Difference between the bacterial composition of the freshwater and seawater environments was identified by MiSeq sequencing analysis.
\end{abstract}

Key Words: Bacterial composition, Freshwater, Seawater, 454 Pyrosequencing, Miseq sequencing

\section{INTRODUCTION}

The ecosphere is dominated by microorganisms (Whitman et al., 1998). The distribution and abundance of bacteria are influenced by ecosystemic and environmental factors (Chapin III et al., 2000). However, the distribution and abundance of environmental microorganisms has not been accurately identified by pre-existing culture methods. It is estimated that less than $0.1 \%$ of bacterial species can be cultivated under laboratory conditions and thus the identification of microorganisms has been limited using culturedependent assays (Torsvik et al., 1990; Torsvik and Øvreås, 2002). Recent advances in molecular microbial ecology provide a compelling approach for detailing the existence of hundreds or thousands of microorganisms in the environment in various numbers and varieties (Giovannoni et al., 1990; Li et al., 2012; Segata et al., 2012). This recent advance

*Received: November 2, 2016 / Revised: December 7, 2016 / Accepted: December 8, 2016

${ }^{\dagger}$ Corresponding author: Yong-Bin Eom. Department of Biomedical Laboratory Science, College of Medical Sciences, Soonchunhyang University, 22, Soonchunhyang-ro, Shinchang-myeon, Asan-si, Chungcheongnam-do 31538, Korea.

Tel: +82-41-530-3039, Fax: +82-41-530-3085, e-mail: omnibin@sch.ac.kr

(C) The Korean Society for Biomedical Laboratory Sciences. All rights reserved.

(C) This is an Open Access article distributed under the terms of the Creative Commons Attribution Non-Commercial License (http://creativecommons.org/licenses/by-nc/3.0/) which permits unrestricted non-commercial use, distribution, and reproduction in any medium, provided the original work is properly cited. 
in research has been called 'Metagenomics'. Metagenomics is the collection of genomes and genes from microbes in their constituent environments; and it enables the characterization of hundreds or even thousands of microorganisms that constitute the microbial community, or microbiome, of an ecosystem (Handelsman et al., 1998). Based on these previous studies, we conducted culture-independent and culturedependent methods in order to compare their results for identical samples. For the identification of cultivable microbes in the sample, the matrix-assisted laser desorption/ ionization time-of-flight (MALDI-TOF) method was used. For microbial identification using the culture-independent method, next-generation sequencing (NGS) was used.

The diversity of microbial communities can be represented by the number of taxa presenting in the microbiota. Theoretically, one would expect to find 70 bacterial taxa per milliliter of sewage; 160 bacterial taxa per milliliter of seawater; and anywhere from 6,400 to 38,000 bacterial taxa per gram of soil. The entire bacterial diversity of the ocean may be likely to exceed $2 \times 10^{6}$ taxa; while a ton of soil could include $4 \times 10^{6}$ different taxa (Curtis et al., 2002). Among those environments which host the most microbiota, water and soil harbors one of the most diverse microbial communities (Weber et al., 2011). Of these bacterial communities, we selected seawater and freshwater, in order to obtain the profiles of seawater and freshwater microbiota in South Korea and compare the composition of the microbial community of seawater with that of freshwater.

The taxonomic composition of the ecosystem was predicted from the diversity patterns of microbiota and the dominance of bacteria according to their respective environment. Several studies have indicated that it is possible to predict the taxonomic composition of bacterial communities (Chong et al., 2012; Suh et al., 2015; Tinta et al., 2015). Culture-independent methods, which allows for the analysis of microbiota that are recalcitrant to cultivation, has enabled researchers to understand microbial communities since the first studies by Stahl et al. in the 1980s (Stahl et al., 1984; Stahl, 1985). For the culture-independent analysis (highthroughput sequencing), the $16 \mathrm{~S}$ ribosomal RNA (rRNA) gene of microbes encoding the small subunit of the bacterial ribosome was used. The $16 \mathrm{~S}$ rRNA gene is common among prokaryotes but is not present in eukaryotes. The gene contains species-specific variable regions that are useful for identifying phylogenetic relationships (Amann et al., 1995). The samples were amplified by $16 \mathrm{~S}$ rRNA gene PCR. Then, PCR products were analyzed by use of high-throughput sequencing (NGS). NGS technologies, such as the Roche 454 and Illumina et al., have been cornerstones of this sequencing (Margulies et al., 2005).

In this study, we (1) investigated microbial analysis of the same samples using Illumina MiSeq and Roche 454 Pyrosequencing, in order to assess which one is the most capable of analyzing a more diverse range of microbiota; (2) compared the bacterial composition of culture-dependent and culture-independent methods for analyzing the same samples. We undertook freshwater and seawater sampling of the microbial community at the Han River and at Mallipo. The determination of differences in type and frequency of the microbiota related to freshwater and seawater contributes to our knowledge of the distribution of marine and freshwater microbial biogeography and provides a baseline for further studies.

\section{MATERIALS AND METHODS}

\section{Study sites}

Freshwater from the Han River $\left(37^{\circ} 31^{\prime} \mathrm{N}, 127^{\circ} 03^{\prime} \mathrm{E}\right)$ was collected for experimentation. Seawater was collected from Mallipo ( $\left.36^{\circ} 47^{\prime} \mathrm{N}, 126^{\circ} 08^{\prime} \mathrm{E}\right)$. Both water samples from Han River and Mallipo were collected immediately in a sterile bottle. The location chosen for the collection of freshwater was in the middle part of the river (Fig. 1). The study was conducted during from December 2015 to January 2016.

\section{Culture-dependent approach}

Freshwater and seawater $(35 \mathrm{ml})$ were centrifuged at $15,000 \mathrm{rpm}$ for $30 \mathrm{~min}$ and the supernatant was discarded. Then, $200 \mu \mathrm{l}$ of water pellet was inoculated in the blood agar plate, and the sea water pellet was cultured in nutrient agar (Difco, Detroit, US) with $1 \% \mathrm{NaCl}$ solution. The water medium samples were cultured for $24 \mathrm{~h}$ at $37^{\circ} \mathrm{C}$. The MALDI-TOF target plate was inoculated by picking a 


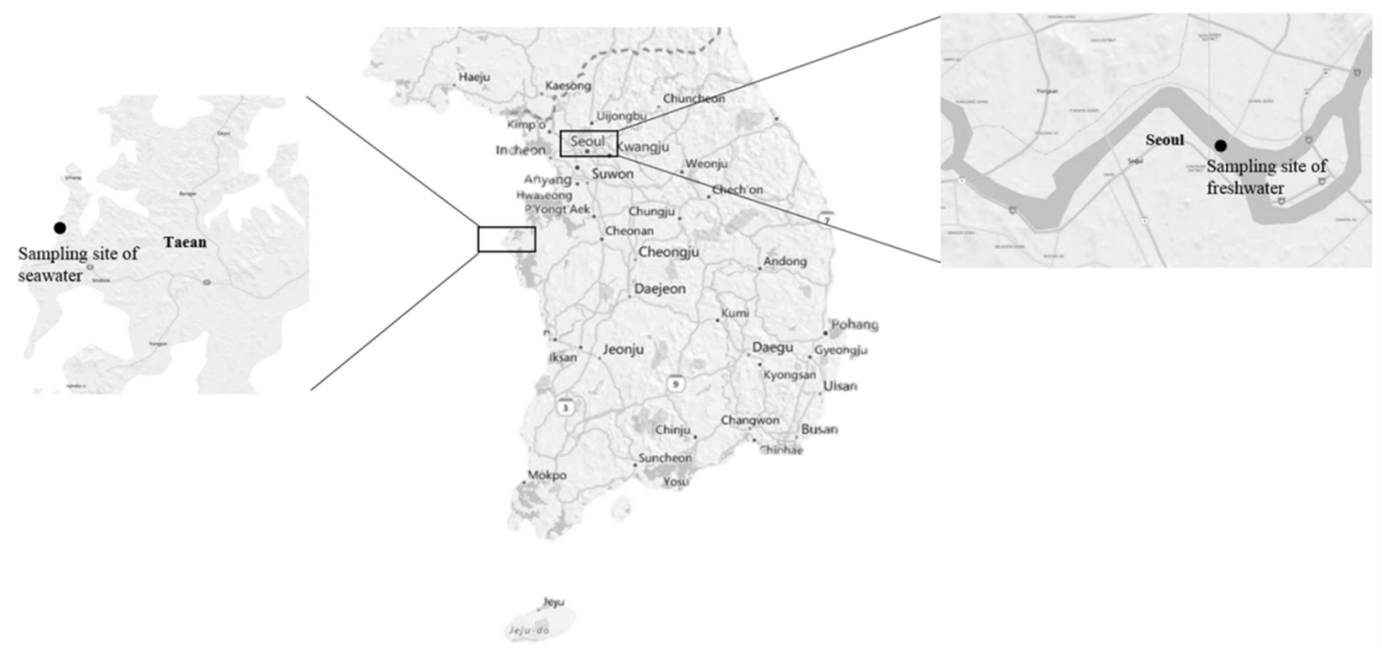

Fig. 1. Maps for the sampling sites in the freshwater and seawater of South Korea. This maps shows the sampling locations in the freshwater $\left(37^{\circ} 31^{\prime} \mathrm{N}, 127^{\circ} 03^{\prime} \mathrm{E}\right)$ and seawater $\left(36^{\circ} 47^{\prime} \mathrm{N}, 126^{\circ} 08^{\prime} \mathrm{E}\right)$. Freshwater and seawater samples were collected in December and January 2015.

freshly-grown colony (grown overnight) with the tip of a sterile toothpick and then smearing the specimen directly onto a thin film. A thin smear of bacteria on the target plate was overlaid with $1 \mu \mathrm{l}$ of a MALDI matrix solution containing alpha-cyano-4-hydroxycinnamic acid in 50\% acetonitrile with $2.5 \%$ tri-fluoracetic-acid. Then, the matrix-sample was crystallized through air-drying at room temperature for $5 \mathrm{~min}$ before testing. The MALDI-TOF MS analysis was conducted via Microflex LT (Bruker Daltonics, GmbH, Bremen, Germany) equipment. FlexControl ${ }^{\mathrm{TM}}$ software was used to calculate and process the analytical database. The spectra obtained were classified using Bruker MALDI Biotyper v2.0 software (Bruker Daltonik GmbH, Germany). For the identification approach, measured mass spectra ranging from 2,000 Da to 20,000 Da was used. The similarity of patterns to the representative species in the database was represented as a log score. The results were categorized as follows: a score of $<1.7$, no reliable identification; a score between 1.7 and 1.9, identification at the genus level; and a score $\geq 2.0$, species identification.

\section{Culture-independent approach}

For DNA extraction, freshwater and seawater samples $(35 \mathrm{ml})$ were centrifuged at $15,000 \mathrm{rpm}$ for $30 \mathrm{~min}$ and supernatant was discarded. DNA was extracted from the pellet using a QIAamp DNA minikit (Qiagen, Valencia, CA, USA). The manufacturer's instructions regarding tissue protocol were followed.

For MiSeq sequencing analysis, PCR amplification was performed using primer targeting the V3 to V4 regions of the 16S rRNA gene of extracted DNA. For bacterial amplification, primers were 341F (5'-TCGTCGGCAGCGTCAGATGTGTATAAGAGACAG-CCTACGGGNGGCWGCAG-3'; the underlined sequence indicates the target region primer; 'AGATGTGTATAAGAGACAG' indicates the adaptor sequence) and 805R (5'-GTCTCGTGGGCTCGG-AGATGTGTATAAGAGACAG-GACTACHVGGG -TATCTAATCC-3'; the underlined sequence indicates the target regions; 'GATGTGTATAAGAGACAG' is the adaptor sequence) (Fadrosh et al., 2014). The PCR reaction mixture included 2.5 $\mu$ of 10X Ex Taq Buffer (Takara Bio, Shiga, Japan), $2.5 \mu \mathrm{l}$ of dNTPs $(2.5 \mathrm{mM}), 1 \mu \mathrm{l}$ of each of the primers ( $10 \mathrm{pmole} / \mu \mathrm{l}), 0.25 \mu \mathrm{l}$ of Taq DNA polymerase ( 5 $\mathrm{U} / \mu \mathrm{l}$; Takara Bio, Shiga, Japan), and $2 \mu \mathrm{l}$ of extracted DNA, in a final total volume of $25 \mu \mathrm{l}$. The amplifications were carried out using an initial denaturation at $95^{\circ} \mathrm{C}$ for $3 \mathrm{~min}$, followed by 25 cycles of denaturation at $95^{\circ} \mathrm{C}$ for $30 \mathrm{sec}$, primer annealing at $55^{\circ} \mathrm{C}$ for $30 \mathrm{sec}$, and extension at $72^{\circ} \mathrm{C}$ for $30 \mathrm{sec}$, with a final elongation at $72^{\circ} \mathrm{C}$ for $5 \mathrm{~min}$. PCR products were confirmed using $1 \%$ agarose gel electropho- 
resis with a 0.5X TAE buffer, and they were visualized using a Gel Doc system (BioRad, Hercules, CA, USA). The PCR product was used as a template in the second PCR. For the second PCR, index primer was Index i5 (AATGATACGGCGACCACCGAGATCTACAC-CTCTCTAT-TCGTCGGCAGCGTC) and Index i7 (CAAGCAGAAGACGGCATACGAGAT-TCGCCTT-AGTCTCGTGGGCTCGG). The PCR reaction mixture conditions were performed as above. Equal concentrations of PCR products were pooled together and short fragments (non-target products) were removed using an Ampure bead kit (Agencourt Bioscience, Beverly, MA, USA). Final DNA libraries were quantified by Quanti-iT PicoGreen dsDNA Assay kit (Invitrogen). Quality and product size were assessed on a Bioanalyzer 2100 (Agilent, Palo Alto, CA, USA) using a DNA 7500 chip. Mixed amplicons were used for emulsion PCR and deposited on Picotiter plates. Sequencing was carried by Chunlab, Inc. (Seoul, Korea) using an Illumina MiSeq sequencing System.

For pyrosequencing, PCR amplification was performed using primers targeting the $\mathrm{V} 1$ to $\mathrm{V} 3$ regions of the $16 \mathrm{~S}$ rRNA gene of extracted DNA. For bacterial amplification, the barcoded primers were 27F (5'-CCTATCCCCTGTGTGCCTTGGCAGTC-TCAG-AC-GAGTTTGATCMTGGCTCAG-3'; the underlined sequence indicates the target region primer; 'TCAG' indicates the key sequence; ' $A C$ ' is the linker sequence) and 518R (5'-CCATCTCATCCCTGCGTGTCTCCGAC-TCAG-X-AC-WTTACCGCGGCTGCTGG-3') (http:/oklbb.ezbiocloud.net/content/1001). The amplifications were performed using the same cycling method of MiSeq sequencing. The amplified products were purified with a MinElute PCR purification kit (Qiagen, Valencia, CA, USA) according to the manufacturer's instructions. The concentration and purity of the resulting DNA were measured with a BioDrop $\mu$ LITE (BioDrop, Cambridge, UK). Short fragments (non-target products) of purified products were pooled together and removed as same method from MiSeq sequencing. Quality and product size were assessed on a Bioanalyzer 2100 (Agilent, Palo Alto, CA, USA) using a DNA 7500 chip. Mixed amplicons were used for emulsion PCR and deposited on Picotiter plates. Sequencing was carried out at Chunlab, Inc. (Seoul, Korea) using a 454 GS FLX titanium next- generation sequencing (NGS) system (Roche, Branford, CT, USA) according to the manufacturer's instructions.

For next-generation sequencing analysis, obtained reads started with a quality check and trimming of low-quality scores (defined as average scores of $<25$ ) by Trimmomatic 0.32. After the quality-control pass, forward and reverse reads were paired using the PANDAseq v.2.9 with default parameters. The taxonomic classification of each read was assigned based on the EzTaxon-e database (http://eztaxone.ezbiocloud.net) (Kim et al., 2012), which contains the 16S rRNA gene sequence of type strains that have valid published names; and the representative species level phylotypes of either cultured or uncultured entries in the GenBank database with complete hierarchical taxonomic classification (levels ranging from the phylum to the species). The richness and diversity of the samples were determined by Chaol estimation and Shannon diversity index at the 3\% distance. Random sub sampling was conducted to equalize the read size of samples; for comparing different read sizes among the samples. The overall phylogenetic distance between communities was estimated using Fast UniFrac (Hamady et al., 2010). To compare Operational Taxonomic Units (OTUs) between samples, shared OTUs were obtained with the CLcommunity program's Taxon XOR by CD-HIT analysis (Chunlab Inc.).

\section{RESULTS}

\section{Culture-dependent analysis of water}

After cultivation of freshwater and seawater, the bacterial species were identified by using MALDI-TOF MS. Abundant genera in the freshwater and seawater samples were Acinetobacter johnsonii, Bacillus species (including Bacillus pumilus, Bacillus cereus, Bacillus megaterium, Bacillus muralis, Bacillus mycoides; and Bacillus licheniformis), Staphylococcus hominis, Micrococcus luteus, Citrobacter freundii and Raoultella ornithinolytica (Fig. 2). MALDITOF MS analysis found no differences between the cultivated microbiota of freshwater and seawater. 


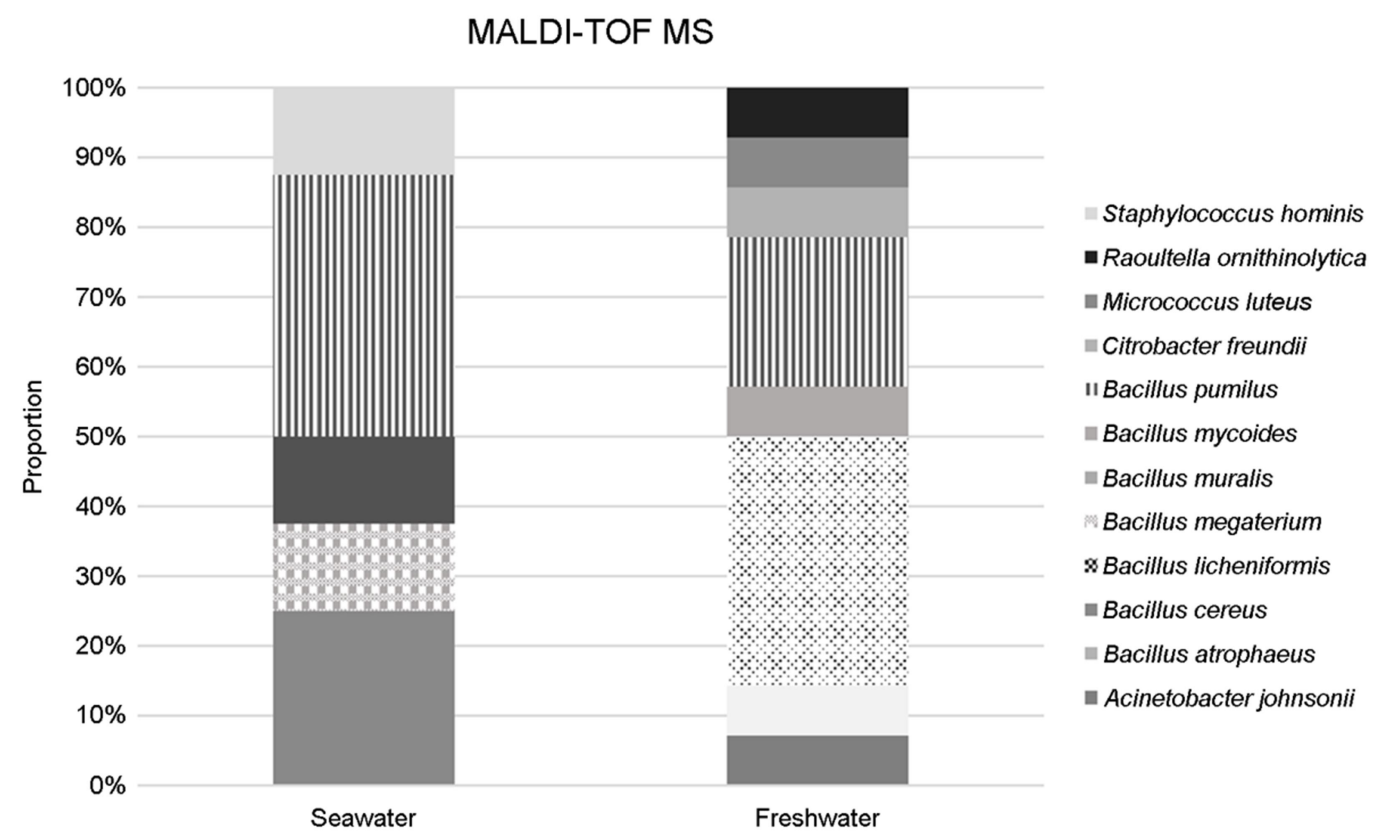

Fig. 2. Relative proportion of microbiota in freshwater and seawater analyzed by MALDI-TOF MS.

Table 1. Numbers of sequences, OTUs (97\%) and diversity indexes of microbiota from freshwater and seawater

\begin{tabular}{lccccc}
\hline \hline \multirow{2}{*}{ Index } & \multicolumn{2}{c}{ Freshwater } & & \multicolumn{2}{c}{ Seawater } \\
\cline { 2 - 3 } \cline { 5 - 6 } \cline { 5 - 6 } No. of Seq & Pyrosequencing & MiSeq & & Pyrosequencing & MiSeq \\
OTUs & 7155 & 17009 & 6030 & 12518 & 6063 \\
Ace & 891 & 77347.07 & & 1237 & 60910.81 \\
Chao1 & 1544.20 & 39007.02 & & 1938.38 & 37069.97 \\
Shannon & 1285.35 & 7.33 & & 5.22 & 7.15 \\
\hline
\end{tabular}

\section{Diversity and composition of bacterial communities associated with freshwater and seawater}

To determine the distribution and composition of microbiota in the water, samples of Han River freshwater and Mallipo seawater were collected (Fig. 1). NGS (454 Pyrosequencing and MiSeq) was performed on the PCR amplicons generated by the pooled DNA from each site. We obtained a total of 61,231 raw sequences. After trimming and quality control, we obtained the following sequences from freshwater and seawater: 7155 and 12518 in pyrosequencing, and 17009 and 17425 in MiSeq sequencing, respectively
(Table 1). The reads from freshwater and seawater were taxonomically clustered into $97 \%$ sequences identity. The number of OTUs in freshwater and seawater analyzed with pyrosequencing were 891 and 1237 (OTUs), respectively. The number of freshwater and seawater OTUs obtained from MiSeq sequencing were 6030 and 6063 (OTUs), respectively. Based on the nonparametric Chao1, Ace, and Shannon index, the known richness of the entire microbial community was identified as being highest in freshwater analyzed with MiSeq sequencing, which showed more bacterial diversity than other samples. Also, the diversity and richness index of the freshwater and seawater samples was 
A

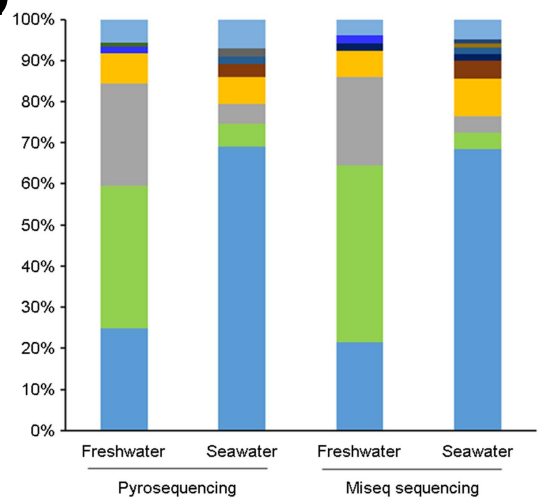

B

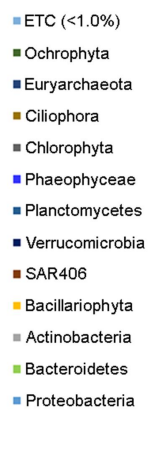

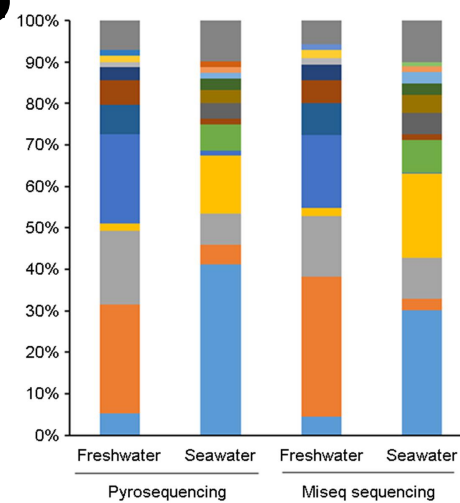

| ETC (<1.0\%)

- Fragilariophyceae

- Verrucomicrobiae

- Cytophagia

- Bacillariophyceae

- Acidimicrobiia

- Mediophyceae

- Sphingobacteria

- Actinobacteria_c

- Betaproteobacteria

- Alphaproteobacteria
" Mamiellophyceae

" Litostomatea

- Ectocarpales_c

= Planctomycetacia

- MarineActino_c

- SAR406_c

- Coscinodiscophyceae

- Deltaproteobacteria

- Gammaproteobacteria

- Flavobacteria

Fig. 3. Taxonomic classification of microbial reads retrieved from different environment water masses into Phylum (A) and Class (B) levels using the EzTaxon database. Display with ration of the abundance, using an EzTaxon database. The names related to the each color appear besides the figure.

higher as found by MiSeq sequencing than as found by pyrosequencing. These data suggest that the diversity and richness index of the microbiota associated with freshwater and seawater in the culture-independent method of MiSeq is higher than that of pyrosequencing.

\section{Bacterial communities of freshwater and seawater}

Illumina MiSeq sequencing analysis and Pyrosequencing was performed to determine the microbiota distribution and composition in freshwater and seawater. These data presented a diversity and richness of the bacterial communities of the associated water samples. We investigated the relative abundance and diversity of the microbial communities in the water samples. To evaluate the bacterial community's composition and distribution, the 16S rRNA sequences were classified at the phylum and family levels (Fig. 3). The total qualified reads could be assigned to bacterial phyla in each sample. The bacterial communities were dominated by Proteobacteria, followed by Bacteroidetes, Actinobacteria, and Bacillariophyta (Fig. 3A). The phylum Proteobacteria (Alpha, Beta, and Gamma) were more abundant in the seawater than in the freshwater samples, accounting for $68.44 \%$ of the community in the seawater.

Further classification to the class level indicated that bacterial communities varied between freshwater and seawater (Fig. 3B). For example, freshwater microbiota were commonly dominated by three classes - Flavobacteria, Actino- bacteria and Betaproteobacteria with a majority of the reads belonging to the phyla Bacteroidetes, Actinobacteria, and Proteobacteria, respectively. Comparatively, in the case of seawater, the microbiota of seawater contained varying proportions of Proteobacteria. Seawater microbiota represents dominance by three classes-Alphaproteobacteria, Gammaproteobacteria, and Betaproteobacteria. In addition, SAR406 and MarineActino in the phyla SAR406 and Actinobacteria, respectively, were only observed in seawater; whereas Alphaproteobacteria and Flavobacteria were represented in all water samples. However, the proportion of Flavobacteria account for a marked percentage of the bacterial communities in freshwater.

\section{Composition of freshwater- and seawater-associated bacterial communities}

Based on the bacterial diversity index and MiSeq sequencing's superior ability to identify members of the bacterial community at the phylum and class level, MiSeq sequencing was selected as the subsequent tool of choice for further classification principally because that sequencing platform had led to the identification of diverse microbiota. As analyzed with MiSeq sequencing, further classification at the genus level found that Flavobacterium, and Planktophila were predominant in the freshwater, while no sequence belonging to the genus Planktophila was detected for the seawater (Fig. 4). By contrast, EU801223 was found to be 

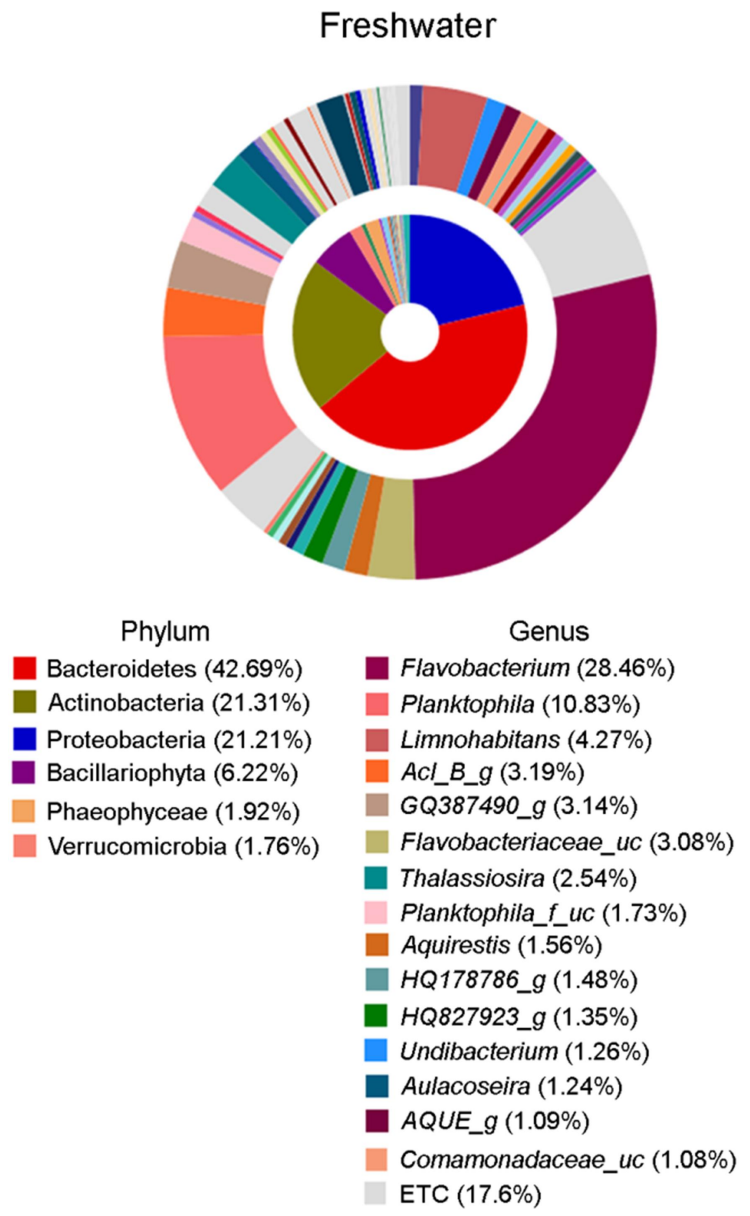

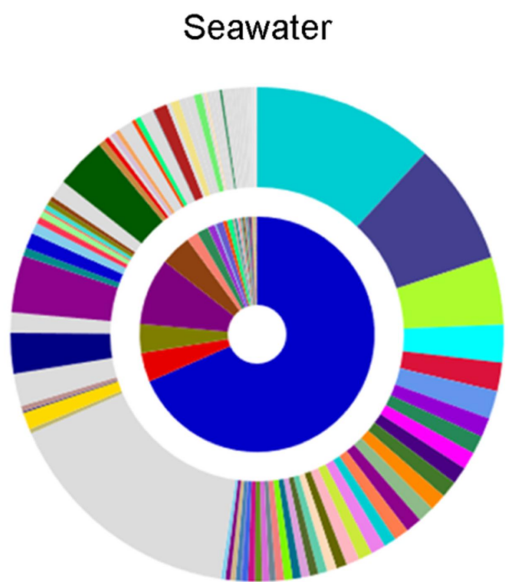

Phylum

Proteobacteria (68.44\%)

Bacillariophyta (9.17\%)

SAR406 (4.3\%)

Bacteroidetes $(4.0 \%)$

Actinobacteria (3.98\%)

Verrucomicrobia (1.61\%)

Plantomycetes $(1.56 \%)$

Ciliophora (1.0\%)

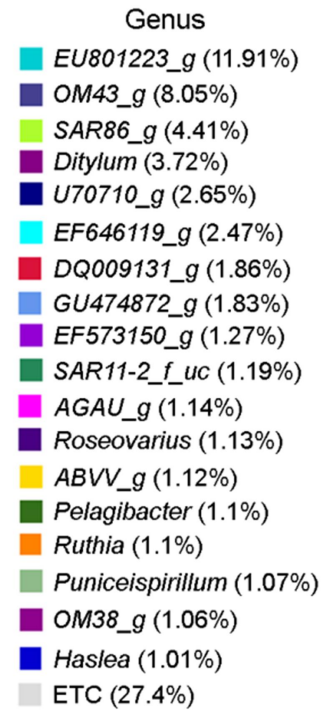

Fig. 4. Bacterial composition profiles. Each bacterial composition of both freshwater and seawater (Inner area: Phylum, Outer area: Genus) Level of phyla and genera with more than $1 \%$ of their proportion were represented. These genera group of freshwater and seawater indicates difference between environmental samples.

a dominant genus among the bacterial community in seawater, although not being present in freshwater. The bacterial communities of freshwater and seawater were significantly different.

\section{DISCUSSION}

We confirmed the identity of the aquatic microorganisms that could be grown under laboratory conditions using cultivation and the MALDI-TOF. We were able to obtain the genera including the genus Bacillus, Micrococcus, and Citrobacter through MALDI-TOF analysis, but could not identify these genus via NGS (Fig. 2). On the other hand, biodiversity at the genus level as revealed by MALDI-TOF analysis was lower than that shown by the MiSeq data. A considerably more diverse numbers of microorganisms have been found when using culture-independent methods as compared to cultivation methods. Relatively more diverse microbiota has was found when using culture-independent methods compared to cultivation methods. The MALDI-TOF MS studies found that the genus Bacillus dominated. Typically, bacteria that thrive in artificial environments can be cultivated in media (Fredricks, 2001). These results were generally in line with former associative analyses regarding comparisons of 
cultivation with non-culture-based methods (Madsen et al., 2015). Although species such as Bacillus pumilus, Micrococcus luteus, and Citrobacter freundii could be found in the water, the species obtained by MALDI-TOF failed to identify the actual differences between species present in freshwater versus seawater.

Further examination involved analysis of both freshwater and seawater samples using the non-cultured method-NGS (454 Pyrosequencing and MiSeq sequencing). We wanted to select the best form of NGS sequencing, which is an effective approach which yields the identification of diverse bacterial communities. Therefore, we generated amplicon of the 16S rRNA gene and performed both 454 Pyrosequencing and MiSeq sequencing. The number of OTUs detected in MiSeq sequencing was more abundant than those yielded by pyrosequencing of the same samples of freshwater and seawater (Table 1). The diversity estimates yielded by analysis via MiSeq sequencing, Shannon, Chaol and Ace, were also higher than were those in samples analyzed with Pyrosequencing. Even in assessing the bacterial communities of the same samples, the results of diversity indexing were markedly different depending on the form of NGS sequencing used. These results seem to be due to a difference in method of analysis. Because the 454 pyrosequencing produces longer read lengths than do competing technologies (from V1 to V3), most investigators prefer to select 454 pyrosequencing as their method of choice. However, while pyrosequencing was capable of producing longer reads lengths, this sequencing produces systemic errors associated with insertions and deletions errors which must be removed or corrected using time-consuming and intensive software before further analysis can be performed (Huse et al., 2007; Quince et al., 2009; Huse et al., 2010; Reeder and Knight, 2010). By contrast, the Illumina MiSeq sequencing method has a lower per-base error rate as compared with 454 pyrosequencing (Loman et al., 2012).

To assess the differences in the microbial communities of freshwater and seawater, bacterial composition was distinguished in contrast with the results of the cultured method. Freshwater and seawater samples were further analyzed in terms of bacterial composition. The dominant phyla in all the water samples were Proteobacteria, Bacteroidetes, and
Actinobacteria. These results were similar to those shown by previous studies of the microbial communities in seawater (Bolhuis and Stal, 2011; Suh et al., 2014; Suh et al., 2015). Representative freshwater bacteria such as the classes Flavobacteria, Betaproteobacteria, and Actinobacteria classes were detected in the Han River sample. Also, a predominance in the occurrence of certain marine bacteria including the classes Alphaproteobacteria, Gammaproteobacteria, and Betaproteobacteria were found in the Mallipo seawater sample.

Based on the bacterial community and diversity index, the results related to the MiSeq sequencing represents findings more diverse than those yielded by 454 pyrosequencing. Therefore, we performed further classificatory analysis at the genus level, in order to minutely compare freshwater microbiota with seawater microbiota. At the genus level, Flavobacterium and Planktophila were identified as being genera unique to the freshwater sample; whereas EU801223, OM43, and SAR86 were uniquely found only in the seawater sample. Unlike the results of the culture-dependent method, the bacterial composition of culture-independent method showed clear differences between the populations of the freshwater and seawater environment. Environmental factors can affect the distribution and the abundance of bacteria.

This study has some limitations, one of which was that we could not confirm the degree to which bacterial composition may have been related to diverse environmental factors such as temperature, salinity, $\mathrm{pH}$, and oxygen levels that could have potentially changed the water-associated microbial communities. However, although the present study has the above-noted limitation, we demonstrated that Illumina MiSeq sequencing of $16 \mathrm{~S}$ rRNA is a more effective approach as compared to other options in that it can analyze to a greater degree of specificity the composition of diverse microbial populations and can obtain profiling data regarding the microbial community in relation to that community's ecosystem. Of special importance, this study offers the potential of enhancing our comprehension of the marine and freshwater ecosystems of South Korea. Understanding the patterns of microbial composition and diversity is a necessary first step in going on to assess the systemic effects of specific microbiota on their respective freshwater or marine ecosystems. The present investigation was a pilot study for 
establishing which method would yield the highest-quality findings in terms of specificity. Thus, a greater degree of environmental factors relative to water should be examined in further studies. A diagnosis of drowning remains one of the challenge for forensic medicine because the use of diatom as a test of drowning diagnosis has some limitations (Piette et al., 2006). Although diatom are not present in all water sources or may not be abundant, aquatic microbiota diverse between the habitats as shown in the study. Therefore, we are also currently in the process of applying the microbial community profiling of freshwater and seawater, as obtained at this time, to the forensic sciences.

\section{Acknowledgments}

This study was supported by the Soonchunhyang University Research Fund; the Basic Science Research Program, through the National Research Foundation of Korea, funded by the Ministry of Education (NRF-2015R1D1A1A02061773); and the Laboratory Safety Management Program, through the National Research Foundation of Korea, funded by the Ministry of Science, ICT and Future Planning (NRF-2016H1D7A2020904).

\section{Conflict of interest}

The authors declare no conflicts of interests in the publication of this article.

\section{REFERENCES}

Amann RI, Ludwig W, Schleifer K-H. Phylogenetic identification and in situ detection of individual microbial cells without cultivation. Microbiol Rev. 1995. 59: 143-169.

Bolhuis H, Stal LJ. Analysis of bacterial and archaeal diversity in coastal microbial mats using massive parallel 16S rRNA gene tag sequencing. ISME J. 2011. 5: 1701-1712.

Chapin III FS, Zavaleta ES, Eviner VT, Naylor RL, Vitousek PM, Reynolds HL, Hooper DU, Lavorel S, Sala OE, Hobbie SE. Consequences of changing biodiversity. Nature. 2000. 405: 234-242.

Chong C, Pearce D, Convey P, Tan I. The identification of environmental parameters which could influence soil bacterial community composition on the Antarctic Peninsula-a statistical approach. Antarctic Science. 2012. 24: 249-258.
Curtis TP, Sloan WT, Scannell JW. Estimating prokaryotic diver sity and its limits. Proceedings of the National Academy of Sciences. 2002. 99: 10494-10499.

Fadrosh DW, Ma B, Gajer P, Sengamalay N, Ott S, Brotman RM, Ravel J. An improved dual-indexing approach for multiplexed 16S rRNA gene sequencing on the Illumina MiSeq platform. Microbiome. 2014. 2: 6.

Fredricks DN. Microbial ecology of human skin in health and disease. J Investig Dermatol Symp Proc. 2001. 6: 167-169.

Giovannoni SJ, Britschgi TB, Moyer CL, Field KG. Genetic diversity in Sargasso sea bacterioplankton. Nature. 1990. 345: 60 -63 .

Hamady M, Lozupone C, Knight R. Fast UniFrac: facilitating highthroughput phylogenetic analyses of microbial communities including analysis of pyrosequencing and PhyloChip data. The ISME Journal. 2010. 4: 17-27.

Handelsman J, Rondon MR, Brady SF, Clardy J, Goodman RM. Molecular biological access to the chemistry of unknown soil microbes: a new frontier for natural products. Chem Biol. 1998. 5: R245-249.

Huse SM, Huber JA, Morrison HG, Sogin ML, Welch DM. Accuracy and quality of massively parallel DNA pyrosequencing. Genome Biology. 2007. 8: 1.

Huse SM, Huber JA, Morrison HG, Sogin ML, Welch DM. Ironing out the wrinkles in the rare biosphere through improved OTU clustering. Environmental Microbiology. 2010. 12: 1889-1898.

Kim OS, Cho YJ, Lee K, Yoon SH, Kim M, Na H, Park SC, Jeon YS, Lee JH, Yi H, Won S, Chun J. Introducing EzTaxon-e: a prokaryotic 16S rRNA gene sequence database with phylotypes that represent uncultured species. Int J Syst Evol Microbiol 2012. 62: 716-721

Li K, Bihan M, Yooseph S, Methe BA. Analyses of the microbial diversity across the human microbiome. PLoS ONE. 2012. 7: e32118.

Loman NJ, Misra RV, Dallman TJ, Constantinidou C, Gharbia SE, Wain J, Pallen MJ. Performance comparison of benchtop high-throughput sequencing platforms. Nature Biotechnology. 2012. 30: 434-439.

Madsen AM, Zervas A, Tendal K, Nielsen JL. Nielsen. Microbial diversity in bioaerosol samples causing ODTS compared to reference bioaerosol samples as measured using Illumina sequencing and MALDI-TOF. Environ Res. 2015. 140: 255-267.

Margulies M, Egholm M, Altman WE, Attiya S, Bader JS, Bemben LA, Berka J, Braverman MS, Chen Y-J, Chen Z. Genome sequencing in microfabricated high-density picolitre reactors. 
Nature. 2005. 437: 376-380.

Quince C, Lanzén A, Curtis TP, Davenport RJ, Hall N, Head IM, Read LF, Sloan WT. Accurate determination of microbial diversity from 454 pyrosequencing data. Nat Methods. 2009. 6: 639 .

Piette MH, De Letter EA. Drowning: still a difficult autopsy diagnosis. Forensic Science International. 2006. 163: 1-9.

Reeder J, Knight R. Rapid denoising of pyrosequencing amplicon data: exploiting the rank-abundance distribution. Nat Methods. 2010. 7: 668.

Segata N, Waldron L, Ballarini A, Narasimhan V, Jousson O, Huttenhower C. Metagenomic microbial community profiling using unique clade-specific marker genes. Nat Methods. 2012. 9: 811-814.

Stahl DA, Lane DJ, Olsen GJ, Pace NR. Analysis of hydrothermal vent-associated symbionts by ribosomal RNA sequences. Science. 1984. 224: 409-411.

Suh SS, Park M, Hwang J, Kil EJ, Jung SW, Lee S, Lee TK. Seasonal dynamics of marine microbial community in the South Sea of Korea. PLoS One. 2015. 10: e0131633.

Suh S-S, Park M, Hwang J, Lee S, Moh SH, Park KH, Lee T-K.

Characterization of bacterial communities associated with seasonal water masses from Tongyoung in south sea of Korea. Ocean Science Journal. 2014. 49: 193-200.

Tinta T, Vojvoda J, Mozetič P, Talaber I, Vodopivec M, Malfatti F, Turk V. Bacterial community shift is induced by dynamic environmental parameters in a changing coastal ecosystem (Northern Adriatic, Northeastern Mediterranean Sea)-a 2-year time-series study. Environmental Microbiology. 2015. 17: 3581 -3596 .

Torsvik V, Goksøyr J, Daae FL. High diversity in DNA of soil bacteria. Applied and Environmental Microbiology. 1990. 56: 782-787.

Torsvik V, Ovreas L. Microbial diversity and function in soil: from genes to ecosystems. Current Opinion in Microbiology. 2002. 5: 240-245.

Weber M, Teeling H, Huang S, Waldmann J, Kassabgy M, Fuchs BM, Klindworth A, Klockow C, Wichels A, Gerdts G. Practical application of self-organizing maps to interrelate biodiversity and functional data in NGS-based metagenomics. The ISME journal. 2011. 5: 918-928.

Whitman WB, Coleman DC, Wiebe WJ. Prokaryotes: the unseen majority. Proc Natl Acad Sci. 1998. 95: 6578-6583. 